\title{
HADAMARD SPECTROMETRY FOR THERMOLUMINESCENCE INVESTIGATIONS
}

\author{
II.L. OcZkowski \\ Instilute of Physics, N. Copcrnicus University, Grudziądzka 5, 87-100 Toruń, Poland
}

(Received May 25, 1992)

\begin{abstract}
A Iladamard encoded spectrometcr with a special variable interference filter allows attaining high throughput and sensitivity. In our instrument the interference filter is fixed between condenser lenses. The transmitted wavelength changes continuously along the length of the filter. The multislit cyclic Hadamard mask moves step by step behind the filter to multiplex signal. Some details concerning the data recording, handling and decoding are specific for this technique of investigation of thermoluminescence spectrum.
\end{abstract}

PACS numbers: 07.65.-b, 78.60.Kn

\section{Introduction}

The ability to trap some of excess carricrs cxcitcd by an external source of radiation cnables encrgy to be prescrved in a metastable state. An evolution of the intensity of thermoluminescencc (TL) emission is determined by the complex kinetic processes of thermal release, retrapping and radiant recombination of excess carriers. As a rule, TL is always related to the increase of temperaturc of investigated sample. After excitation, the increase of temperature strongly enhances the process of trap emptying, thus stimulating TL. A competition between the increasing probability of rclease from the trap and the depletion of filled traps and luminescence centres causes a temporary character of TL.

Each sort of trap and cventually associated recombination centre are related to their own kinetic process and to a constituent peak of a glow curve. An cvolution of the spectrum of TL cmission supplics infromation about the type of the active recombination centres and provides fundamental details necessary for the formation of the kinetic band model. Unforlunately, the sum of TL emission is limited and comparatively low. For example, during TL $\mathrm{ZnSe}$ crystal excited up to saturation in $80 \mathrm{~K}$ emits about $10^{13}$ photon $/ \mathrm{cm}^{3}$. Moreover, total light intensity and spectrum depend on time. For these reasons thermoluminescence spectrometry has specific requirements [1]. 
In general, two fundamental methods of spectrometry are used in TL investigations. When a monochromator is used, the entire spectrum is recorded by scanning the dispersion element. An exit slit positioned in a focus plane of spectrum sclects a small portion of dispersed radiation for detection. Since only the rays of determined wavelength and bandwidth pass through the monochromator for measurements and the other are absorbed, this method, applied to the phenomenon with the limited sum of light, is extremely wasteful [2-5]. The same effect is obtained when a filier composition or continuous interference filter is used $[6,7]$.

In the second case, a multichannel or multidetector technique is applied. For any of scveral types of photodetcclor devices (e.g. photodiode array, vidicon, image intensifier and also photograplyy plate) the sensitive clement spreads over an arca of spectrum. The spectrum corresponding to all slit positions is now monitored at once $[8-11]$.

\section{Multislit spectroscopy}

A multiplexed spectrometer contains only one photodetector, but the radiation, which is allowed to impinge at the same time upon a detector, arises from many different parts of the dispersed spectrum. The manner of light mixing is controlled by an array of narrow equidistant, opened or closed slits. This multislit array forms a binary $\{0,1\}$ encoding mask usually placed in the exit (or at entrance) of the focal planc of a conventional spectrometer. The beam intensity incident on detector is measured for many different configurations of mask patterns. Each mask pattern sclects a group of data corresponding to the spectral density of open clements. All selected data are summed up by one detector. This way, the chosen set of spectral data goes through an cncoding multiplex transformation.

The total intensity measured at the detector $y_{i}$ can be written as a sum of the spectral densities $x_{j}$ weighted by a binary factor $a_{i j}$

$$
y_{i}=\sum_{j}^{n} a_{i j} x_{j},
$$

where $i=1,2, \ldots N$ are the pattern numbers and $j=1,2, \ldots n$ numbers of the positions of spectral clements. The coefficients $a_{i j}$ are cither 0 or 1 and correspond to attenuation or transmission.

A proper arrangement of $N$ different masks gives for $n=N$ a desired set of linearly independent equations which allows the calculation of spectral densitics $x_{j}$

$$
x_{j}=\sum_{i}^{n} b_{i j} y_{i}
$$

where $b_{i j}$ are coefficients of the inverse matrix.

A deep theoretical analysis performed mainly in the late 1960's was able to give solutions for several additional questions determining a possibility of wide application of the encoded multiplexing to spectrometry. The basic problems in designing this method are the following: 
(a) For easy implementation, the matrix of $a_{i j}$ coefficients is required to be cyclic. This property allows moving a mask step by step and tremendously simplifies the mask structure. Instcad of $n$ separated masks, each one with different configuration of $n$ elements (open or close slits), the one cyclic mask contains $2 n-1$ elements.

(b) The choice of the optimum mask codcs $a_{i j}$ in order to minimize the influence detector noise on the final result and maximize the signal-to-noise ratio, must be performed. The proper concept to find a solution for this question is based on the theory of digital communications and is related to mathematics of IIadamard matrices. For this reason Decker and IIarwit [12] call this new field IIadamard Transform Spectrometry.

(c) Cramcr's fornulas are not applicable for large set of linear equations (1) therefore a simple algorithm for calculation of spectral densities $x_{i}$ is required.

The details of theoretical analysis are presented in several original papers [12-15], review articles (e.g. [16]) and monograplıs [17-20]. As it was finally shown by Nelson and Fredman [14] in 1970, the proper answers for all these questions lic in IIadamard simplex matrices typically abbreviated as S-matrices. Especially amazing is the simplicity of the decoding algorithm. For $n=N$ the coefficients $a_{i j}$ are transformed to $b_{i j}$ by the following relationships:

$$
\begin{aligned}
& \text { if } a_{i j}=0 \text { then } b_{i j}=-1 / m, \\
& \text { if } a_{i j}=1 \text { then } b_{i j}=+1 / m,
\end{aligned}
$$

where

$$
m=(n+1) / 2 .
$$

In the IIadamard encoding mask for each configuration $m$ slits are open at once and the measurements are repeated $n$ times in order to complete the set of $y_{i}$ values. This leads to the high signal-to-noise ratio of the multislit method. The ratio is $\sqrt{n} / 2$ times higher than for the single-slit scanning.

\section{Hadamard spectrometer}

The main designing assumption of the presented IIadamard spectrometer is an application for measuring spectrally resolved TL. The following preliminary terms were determined:

a) One sequence of a spectrum measurement docs not take more than one minute of time. Ilence for a typical healing rate of the order of $0.1 \mathrm{Ks}^{-1}$ one spectrum docs not cover more than $10 \mathrm{~K}$ of increasing tempcrature.

b) The spectrometer should be compatibile with our already existing arrangements and technique of measurement which was previously described [21].

c) Taking into account the conflict betwecn spectral resolution and brightness, the rather low resolution of $5 \mathrm{~nm}$ is assumed in exchange for high efficiency of light gathering and system compactness.

Due to the last reason the continuous interference filter (Zeiss Jena) with an additional blocking color glass laycr is applied as a dispersion element for the wavelength range of spectrum from 400 to $765 \mathrm{~nm}$. This filter is a rectangular plate 
of $73 \times 18 \mathrm{~mm}^{2}$ size. The transmitted wavelength changes continuously along the length of the filter with the rate of $5 \mathrm{~nm} / \mathrm{mm}$. The active part of the filter is limited by a window of size $63 \times 18 \mathrm{~mm}^{2}$. The fixed filter positions allow choosing two ranges of spectrum, 400 to $715 \mathrm{~nm}$ and 450 to $765 \mathrm{~nm}$. For cone and tilt angles of incident light not exceeding 15 degrees the resolution power is about $5 \mathrm{~nm}$. The average peak transmittance is about 0.2 .

Figure 1 shows the optical configuration of the IIadamard spectrometer. A

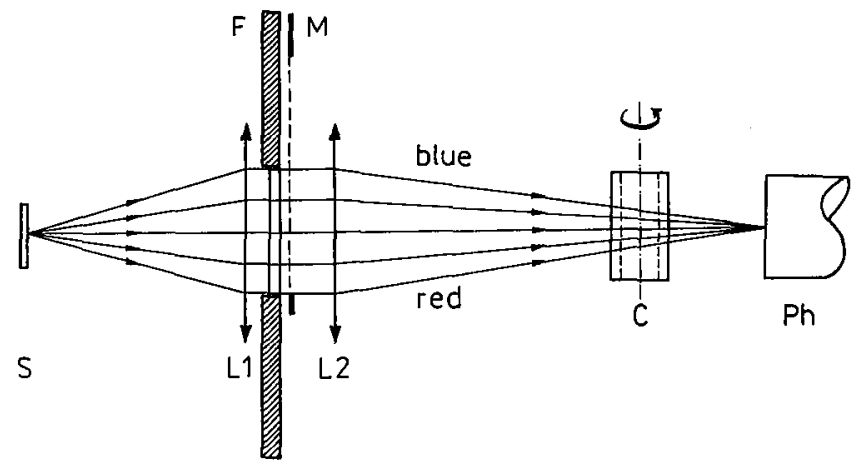

Fig. 1. A schematic diagram showing the operation of the Iladamard spectrometer with the continuous interference filter as a dispersion element and multislit encoding mask; S-sample placed at the input focus of the condenser system, L1, L2 - condenser lenses, $\mathrm{F}$ - window and interference filter, $\mathrm{M}$ - encoding mask, $\mathrm{C}$ - chopper and $\mathrm{Ph}$ - photomultiplier at the output focus of condenscr. There are no input and output slits in the spectrometer presented here.

simple condenser arrangement collects light from the source placed in the input focus, collimates in the filter position and again focuses rays on the detector photocatode. Near the output focus the chopper is position to modulate bcam of light for the phase sensitive detection system.

At a very small distance behind the filter the multislit mask is shifted by the precision screw feeder of $1 \mathrm{~mm}$ pitch. The cyclic mask consists of 125 clements, each of them in shape of opaquc or transparent rectangle $(1 \mathrm{~mm}$ wide, $18 \mathrm{~mm}$ high). For each $1 \mathrm{~mm}$ shift of the mask, a ncw group of 63 rectangles covers the length of the window with the continuous filter and forms a different pattern of open or close slits. For each one of the patterns 32 slits are open at once. IIence, due to the encoding cyclic mask and multiplex tcchnique, the active area of the filter is constantly equal to $32 \times 18 \mathrm{~mm}^{2}=576 \mathrm{~mm}^{2}$ instead of $18 \mathrm{~mm}^{2}$, relevent to the single-slit scanning.

The construction of the encoding cyclic matrix is based on primitive polynomials modulo 2. This method generates matrices having period of $n=2^{k}-1$, where $k$ is the degree of the primitive polynomial. The first $k$ elements of the calculated sequence may be chosen arbitrarily (except not all zero). The other $n-k$ elements of the first row of the encoding cyclic matrix are obtained by a simple algorithm $[14,17]$. The following encoding pattern has been calculated for $n=63$, 
$k=6$ and the all "seed" elements equal oncs:

1111110000010000110001010011110100011100

10010110111011001101010.

Next, the initial 62 elements of this pattern are repeated. Ilence, the pattern of the whole mask consists of 125 elements. The length of the window covers a group of 63 elements of the mask. This way, the succesive moving of the cyclic mask provides 63 patterns each containing 63 encoding elcments. The initial six open ficlds at the beginning of the mask (seed) facilitate the precision of aligning of the mask and window edge.

\section{Measurement system}

The encoded light is modulated by an cylindric, two-aperture chopper. The rotation axis of the chopper is positioned along the dispersed spectrum to perform the modulation of all elements of the spectrum at once. A reference signal generated by this system controls the phase-scnsitive lock-in amplifier. The chopping frequency is around $77 \mathrm{IIz}$. Filtered, encoded and modulated light is detected by the cooled photomultiplier EMI 6203.

The phototube output $(1 \mathrm{M} \Omega)$ is equipped with an integrating filter proper to the chopping frequency (time constant is about $0.01 \mathrm{~s}$ ). An output signal is amplified and smoothed in lock-in amplifier by the electronic band-pass (50-150 IIz) and time constant $(0.1 \mathrm{~s})$ filters. The analog signal from the lock-in output is transformed to TTL level by the voltage-frequency converter with the rate of $10 \mathrm{kHz} / \mathrm{V}$.

During measurements the output signals are monitored and recorded by the multichannel analyser Tristan-1024 (Polon) connected with the small interface/driver system Izolda and a microcomputer. The analyser works as a master in multiscaler mode, switching channels with desired time rate (typically $0.5 \mathrm{~s}$ ). One sequence of spectrum measurement uses 64 channels, where 63 channels collect data for the decoding procedure and the last 64th channel is meaningless, because it corresponds to the reverser of the mask motion.

A channel switching signal from the analyser synchronizes the Izolda, which generates TTL pulses to control the driver of slepping motor. A group of 72 pulses shifts the mask exactly $1 \mathrm{~mm}$ further to the next encoding configuration. The interface offers several additinal options. For example, it is possible to switch two different signals to odd and even channels for simultancous recording a multiplexed spectrum and global TL emission or temperature. But since the TL resolving spectra are usually measured for the linear increase of temperature and this is carefully controlled by a separate set-up [21], regarding masurement efficiency, as a rule, the sample temperature is not registrated by the multichannel analyser.

In order to test, an array of commercial electroluminescence (EL) diodes (red, yellow and green) was used as a light source for the IIadamard spectrometer. This testing set-up was supplied from the low-frequency generator. Figure 2 gives an experimental example of curves registrated by the multichannel analyser; (a) shows the total EL intensity measured without a dispersion element and (b) the record of multiplexed signals for sixtcon sequences. The vertical lines separate 

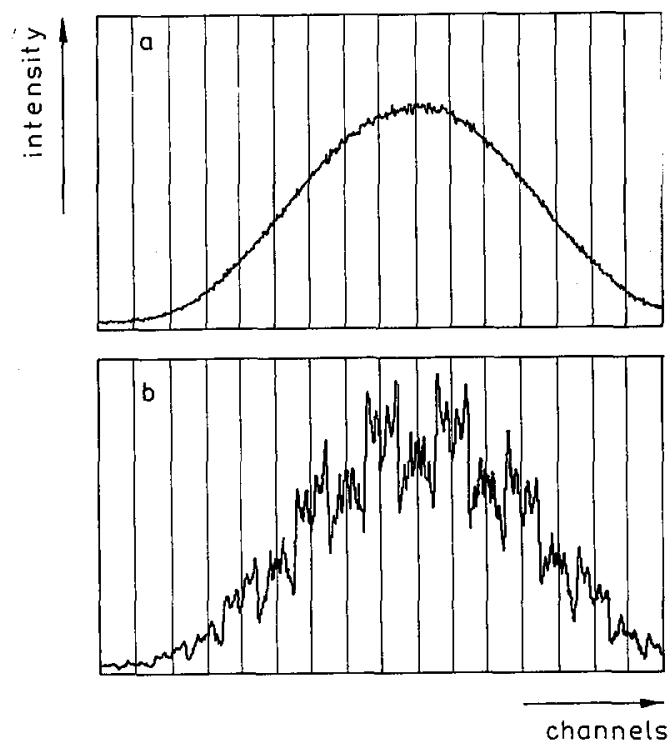

Fig. 2. Two records from the multichannel analyser showing the dependence of the light intensity on time (channels) for testing array of EL diodes supplied from a low-frequency gencrator; (a) the total light intensity, (b) the multiplexed signal for sixtcen sequences (vertical lines).

sequences with the reverse cncoding order. Data recorded by Tristan analyser are transferred via computer and stored as a disk data file. It seems to be interesting to note here that in comparison with the single-slit scanning method an amplitude of variation of the multiplexed signals is significantly lower. This effect appears to be greater for broader band spectra.

\section{Data processing}

The numerical procedure consists of scveral steps of calculations. At first, the data for reverse sequence are transposed and the file is rearranged to make the proper matrix of 63 encoded data for cach scquence.

The next step of data handling is cspecially important for TL emission spectra. Since the light level of TL is not constant in time, the encoded 63 intensities for each sequence are corrected by the cocflicients of linear approximation of light variation. The sums of matrix elements for scquences allow the calculation of averaged rates of the light cvolution. Of course, the independent measurcment of the total TL emission allows performing a direct normalization of encoded data.

Without the correcting treatment the decoding procedure gives the systematic error which is specific for the encoding mask and depends on the light fluctuation and spectrum. The bchaviour of decoding crrors is quite different from the usual spectrometric errors arising from noises, delay time or resolution powcr. After decoding of uncorrected signals even the negative emission densities for some broad regions of the spectrum can be obtained. 


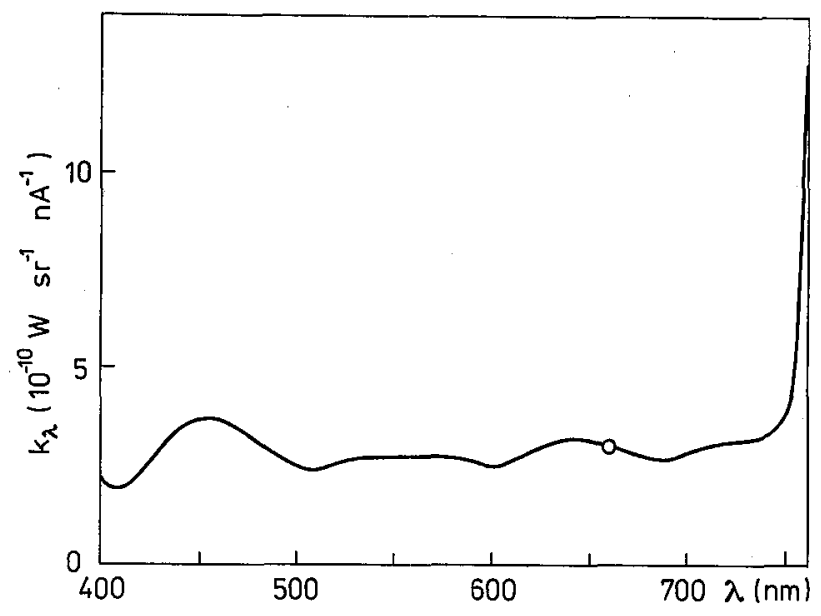

Fig. 3. The spectrum of radiant sensitivity of the whole optical and detecting system of the Iladamard spectrometer.

The corrected data, sequence by sequence, are decoded. The direct method based on formula (2) and relation (3) or any faster algorithm of calculation [14, 20] can be here applied. Next, the results must be calibrated with respect to the spectral response of the whole optical and detecting system. To determine the desired spectral factors the calibrated light source with the known spectrum (blackbody $2854 \mathrm{~K}$ ) was used. It should be mentioned here that the spectral response shown in Fig. 3 is expressed as the absolute radiant sensitivity [ $\left.\mathrm{W} \mathrm{sr}^{-1} \mathrm{nA}^{-1}\right]$. Practically, $0.1 \mathrm{n} \Lambda$ can be assumed as a low limit of detected photomultiplier current (for

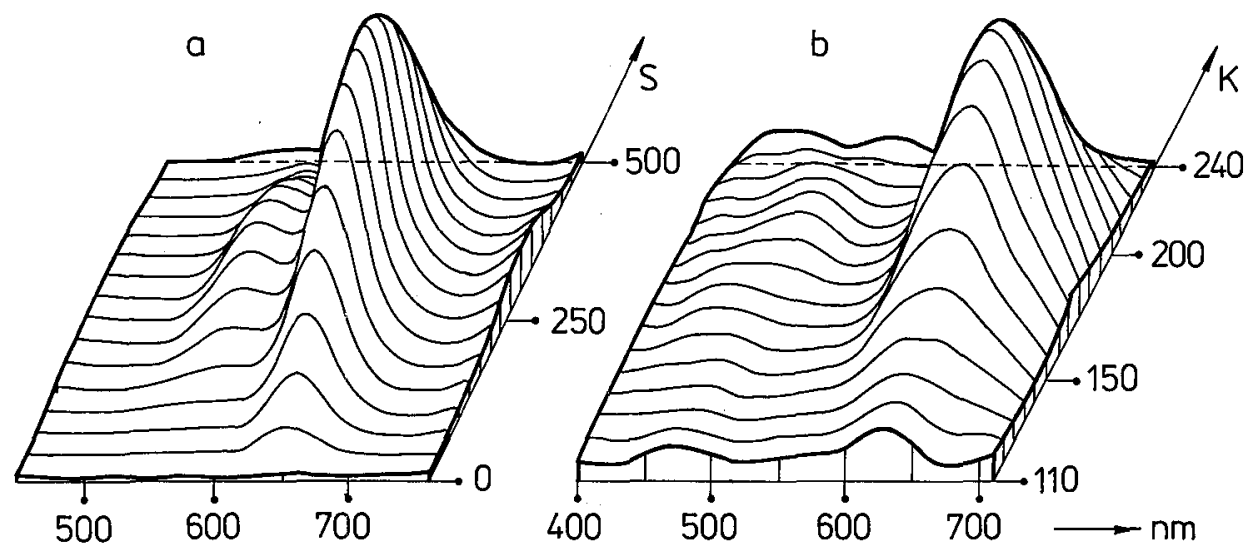

Fig. 4. Spectrally resolved luminescence; (a) the dependence of EL spectrum on time. This plot corresponds to the encoded record in Fig. 2b. (b) The curve (b) shows the temperature resolved spectra of $\mathrm{TL}$ for $\mathrm{ZnSe} \mathrm{Ag}$. 
$0.5 \mathrm{~s} /$ channel). Ilence, the presented curve also allows estimating the low limit of the light intensity which is sufficicnt to record reliable spectra. For $660 \mathrm{~nm}$ this limit corresponds to the intensity of about $10^{8}$ photons $\mathrm{sr}^{-1} \mathrm{~s}^{-1}$. The absolute calibration was performed for $660 \mathrm{~nm}$ by means of the radiometer IP $8334 / 8330 \mathrm{~A}$, photomultiplier and red EL diode.

After the calibrating treatment the matrix elements represent the desired spectral densities. Of course, the place of elements provide additional information concerning the wavelength and time of measurement. The final procedure may include several options like numerical filtering of noise, smoothing, spectral deconvolution and usually graphics program.

Figure 4 gives two examples of spectrally resolved luminescence; (a) shows the time resolved spectra of the steady-state EL emission of diode array. This plot represents the decoded record of data from Fig. 2. The temperature resolved spectra for $\mathrm{ZnSe}: \mathrm{Ag}$ are shown in Fig. 4(b). The investigated crystal was excited at $80 \mathrm{~K}$ with the light of $365 \mathrm{~nm}$ and TL was measured from $110 \mathrm{~K}$ to $240 \mathrm{~K}$ for the heating rate of about $0.153 \mathrm{Ks}^{-1}$.

\section{Conclusions}

The presented here spectrometer has been designed for TL investigation. IIence, the main goal is to maximize the amount of light transferred from a luminescence source to the photomultiplier. The light collection efficiency of differrent spectrometers is well characterized by the optical extend, $G=A \Omega t$, where $A$ is the source area, $\Omega$ the solid angle of an entrance aperture and $t$ the transmittance of the system.

Our spectrometer accepts large area of a sample (say $2 \mathrm{~cm}^{2}$ ). The investigated sample is positioned on the focal length $(11 \mathrm{~cm})$ from the lens collimating light onto the interference filter. Due to the multislit technique the active area of the filter is enriched to $5.76 \mathrm{~cm}^{2}$ which leads to the solid entrance angle of about $0.048 \mathrm{sr}$. Applying $t \approx 0.2$ for transmittance of the system one can obtain the optical extend close to $0.02 \mathrm{sr} \mathrm{cm}^{2}$. It is about one order more than for very bright monochromators with comparable resolution.

A certain incovenience of the encoding spectrometry is inability to reduce the investigated spectral range. The most simple remedy for it is to change the size of the mask. For this reason our next Iladamard spectrometer is equipped with three masks which can be changed casily, namely: $n=63$, shifted by $0.3 \mathrm{~mm}$, 63 by $0.1 \mathrm{~mm}$ and 255 by $0.1 \mathrm{~mm}$. This spectrometer has a prism and is not so bright but allows reaching higher spectral resolution and is specially adjusted for steady-state photoluminescence investigation.

\section{Acknowledgements}

In the course of technical works several specialists gave me a substantial help in construction of many details of the presented apparatus. I am particulary grateful to M. Graczewski, B. Ozdzinski, K. Pancewicz, W. Wenta and Z. Wojtowicz. It is also a great pleasure to extend my warm thanks to members of our 
Institute R. Duda, Dr A. Kowalczyk, J. Kowalski, L. Polakiewicz and II. Schiller. The support for the work was provided by the Ministry of National Education, Grant RZL-15/MEN/90.

\section{References}

[1] S.W.S. McKeever, Thermoluminescence of Solids, Cambridge Univcrsity Press, Cambridge 1985.

[2] A.M. Harris, J.II. Jackson, J. Phys. E 3, 374 (1970).

[3] P.L. Mattern, K. Lengweiler, P.W. Levy, Mod. Geol. 2, 293 (1971).

[1] H.L. Oczkowski, Phys. Slatus Solidi A 68, 199 (1981).

[5] P.D. Townsend, K. Ahmed, P.J. Chandler, S.W.S. McKeever, H.J. Whillow, Radiat. Eff. 72, 245 (1983).

[6] I.K. Bailiff, D.A. Morris, M.J. Aitken, J. Phys. E 10, 1156 (1977).

[7] P. Brovetto, A. Delunas, V. Maxia, G. Spano, C. Cortese Nuovo Cimento D 12, 331 (1990).

[8] M. Castagne, J. Gasiot, J.P. Fillard, J. Phys. E 11, 345 (1978).

[9] A.J. Walton, N.C. Dobenham, Nature 284, 42 (1980).

[10] D.J. Huntley, Nuclear Tracks 14, 27 (1988); J. Lumin. 39, 123 (1988).

[11] A.J. Walton, PACT G, 524 (1982); Journal of the European Study Group on Physical, Chemical and Mathematical Techniques Applied to Archaeology, Council of Europe, Strasbourg 1982.

[12] J.A. Decker, Jr., M. IIarwit, Appl. Opt. 7, 2205 (1968); Appl. Opt. 8, 2552 (1969).

[13] N.J.A. Sloane, T. Fine, P.G. Phillips, M. Harwit, Appl. Opt. 8, 2103 (1969).

[14] E.D. Nclson, M.L. Fredman, J. Opt. Soc. Am. 60, 1661 (1970).

[15] J.A. Decker, Appl. Opt. 10, 510 (1971).

[16] R.M. IIammaker, W.G. Fatelcy, D.C. Tilotta, Spectroscopy 3, 14 (1988); Spectrosc. Int. 1, 10 (1989).

[17] S.W. Golomb (Ed), Digilal Communicalions wilh Space Applications, Prentice-ITall, Englewood Clifs 1964.

[18] P.R. Griffiths (Ed), Transform Techniqucs in Chemisiry, Plenum Press, New York, London 1978.

[19] A.G. Marshall, Biophysical Chemistry, Wilcy, New York 1978.

[20] M. IIarwit, N.J.A. Sloane, IIadamard Transform Optics, Acad. Press, New York 1979.

[21] H.L. Oczkowski, Acta Phys. Pol. A76, 649 (1989). 